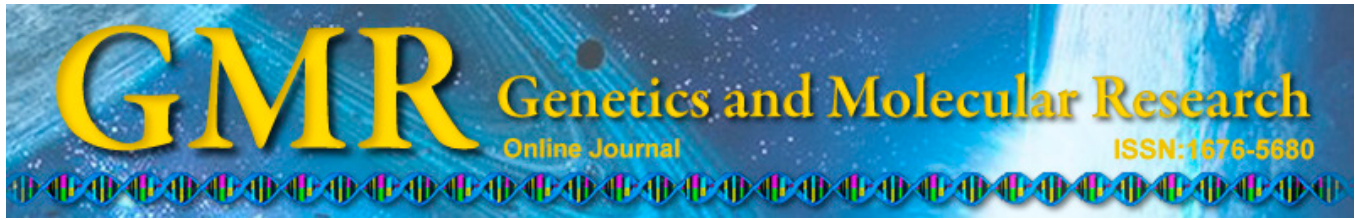

\title{
Postnatal diagnosis of constitutive ring chromosome 13 using both conventional and molecular cytogenetic approaches
}

\author{
L.B. Minasi ${ }^{1,2}$, I.P. Pinto ${ }^{1,2}$, J.G. de Almeida ${ }^{1,2}$, A.V. de Melo ${ }^{1,3}$, \\ D.M.C. Cunha ${ }^{1,2}$, C.L. Ribeiro ${ }^{1}$, G.P. Silva ${ }^{1}$, M.G. Brasil ${ }^{6}$, D.M. Silva ${ }^{1,5}$, \\ C.C. da Silva ${ }^{1,2,4}$ and A.D. da Cruz ${ }^{1,2,3,4}$ \\ ${ }^{1}$ Núcleo de Pesquisas Replicon, Departamento de Biologia, \\ Pontifícia Universidade Católica de Goiás, Goiânia, GO, Brasil \\ ${ }^{2}$ Programa de Pós-Graduação (Mestrado) em Genética, \\ Pontifícia Universidade Católica de Goiás, Goiânia, GO, Brasil \\ ${ }^{3}$ Programa de Pós-Graduação em Biotecnologia e Biodiversidade, \\ Universidade de Brasília, Brasília, DF, Brasil \\ ${ }^{4}$ Laboratório de Citogenética Humana e Genética Molecular, \\ Secretaria do Estado da Saúde de Goiás, Goiânia, GO, Brasil \\ ${ }^{5}$ Programa de Pós-Graduação em Genética e Biologia Molecular, \\ Laboratório de Genética e Biodiversidade, Universidade Federal de Goiás, \\ Goiânia, GO, Brasil \\ ${ }^{6}$ Hospital das Clínicas de Goiânia, Goiânia, GO, Brasil
}

Corresponding author: L.B. Minasi

E-mail:minasilb@gmail.com

Genet. Mol. Res. 14 (1): 1692-1699 (2015)

Received June 17, 2014

Accepted September 24, 2014

Published March 6, 2015

DOI http://dx.doi.org/10.4238/2015.March.6.15

\begin{abstract}
We describe the first postnatal diagnosis of a child from Central Brazil with de novo cytogenetic alterations in $13 \mathrm{q}$ showing malformations of the brain, eyes, distal limbs, and genitourinary tract, and severe intellectual disability. The karyotype was a constitutive 46,XX,r(13)[77]/45,XX,-13[17]/46,XX,idic r(13)[6]. Interphase and metaphase fluorescence in situ hybridization analyses also showed
\end{abstract}


the absence of 13qter and the presence of 13q14.3 in the cells with $\mathrm{r}(13)$, and chromosome microarray analysis detected a $15.39 \mathrm{Mb}$ deletion in chromosome region 13q32.3-q34. This study is intended as the registry of a rare case of chromosomal rearrangement involving chromosome 13 in Central Brazil. Further studies are needed to define whether genetic haploinsufficiency is associated with each major $13 \mathrm{q}$ deletion anomaly.

Key words: 13q deletion; Ring chromosome 13; Complex phenotype; Fluorescence in situ hybridization; Chromosomal microarray analysis; Karyotype

\section{INTRODUCTION}

Deletions of chromosome regions are generally the result of double-stranded chromosome breaks with loss of produced acentric fragments during the next cell division. Such deletions can lead to ring chromosomes. Patients with $\mathrm{r}(13)$ have various phenotypic abnormalities that correspond to specific breakpoints (Uwineza et al., 2013).

Lele et al. (1963) first described a partial deletion of 13q in retinoblastoma patients who also displayed intellectual disabilities and global developmental delays (Huang et al., 2012). The $13 \mathrm{q}$ deletion syndrome leads to phenotypes that include short stature, microcephaly, cerebral cortical malformations, Dandy-Walker malformation (DWM), corpus callosum agenesis, meningocele/encephalocele, neural tube defects, micro-/anophthalmia, cleft lip/palate, lung hypoplasia, heart defects, genital anomalies, and hand abnormalities (Kirchhoff et al., 2009; Chen et al., 2013; Valdes-Miranda et al., 2014). In this study, we used 3 laboratory methodologies to report the first postnatal diagnosis in Central Brazil of a child with cytogenetic abnormalities involving chromosome 13.

\section{MATHERIAL AND METHODS}

\section{Sample preparation and cytogenetic characterization}

The parents of the proband signed informed consent forms approved by the Ethics Committee on Human Research at Pontifícia Universidade Católica de Goiás, under protocol number 1721/2011. Cytogenetic studies were carried out with peripheral blood samples from the patient and her parents using G-banding techniques at a band resolution of $>550$. Shortturn lymphocyte cultures were carried out for the proband and her progenitors following standard procedures (Verma and Babu, 1995). Chromosomal analyses were performed with the IKAROS ${ }^{\circledR}$ software (Metasystems Corporation, Jena, Germany).

Metaphase and interphase fluorescence in situ hybridization (FISH) analyses were performed using 13q14.3 (spectrum red) and 13qter (spectrum green) specific probes according to standard FISH protocols. Chromosomes were counterstained with 4,6-diamino-2-phenyl-indole. Metaphases and interphase nuclei were captured using an epifluorescence microscope (Carl Zeiss, Jena, Germany) and the ISIS ${ }^{\circledR}$ software (Metasystems Corporation, Germany).

Genomic DNA was obtained from peripheral blood from the proband and her parents. Genomic DNA was isolated from whole blood using a QIAamp ${ }^{\circledR}$ DNA Mini kit (Qiagen, Lim- 
burg, Netherlands). The analyses were carried out on the proband and her biological parents to establish whether the DNA rearrangements were de novo or inherited. Total DNA (250ng) for each sample was digested with NspI, ligated, PCR amplified and purified, fragmented, biotin-labeled, and hybridized for use in a GeneChip ${ }^{\mathrm{TM}}$ HD CytoScan Array (Affymetrix, Santa Clara, CA, USA). The array was designed specifically for cytogenetic research, including $\sim 2,696,550$ copy number variation markers, 743,304 single-nucleotide polymorphism markers, and $>1,953,246$ non-polymorphic markers. CEL files obtained by scanning the arrays were analyzed using the Chromosome Analysis Suite software (Affymetrix). Gains and losses that affected a minimum of 50 and 25 markers, respectively, in a $100 \mathrm{~kb}$ length were initially considered.

\section{Case presentation}

A female child was born at 40 weeks gestation to a non-consanguineous Brazilian couple. Her birth weight was $2430 \mathrm{~g}$ and her crown-heel length was $45 \mathrm{~cm}$. Delivery occurred via cesarean section. Malformations in the newborn were noted by the assistant medical staff at birth. At the age of 2 months, the proband underwent surgery for vesico-ureteral reflux. After surgery, the child developed kidney stones. At the age of 8 months, she was diagnosed with low tubular renal function in both kidneys. Her craniofacial dysmorphisms included a high and sloping forehead, hypotelorism, bilateral microphthalmia, deep set eyes with reduction and change in shape of the eyeballs, atrophy of the optic nerves and chiasm, and epicanthal folds. Magnetic nuclear resonance revealed decreased cervical and axial tonus, reduced fontanelle, lack of visualization of the septum pellucidum with persistence of the cavum septum pellucidum and cavum vergae, hippocampal atrophy, absence of falx cerebri, and partial fusion of the thalami with an appearance suggestive of holoprosencephaly. She also had a broad and prominent nasal bridge, arched upper lip, hands with elongated fingers and disproportionate size, and heart problems.

At the age of 6 years, she weighed $16.9 \mathrm{~kg}$, and was $1.06 \mathrm{~m}$ tall. Physical examination revealed severe delayed psychomotor development and intellectual disability. No history of birth defects in the family was reported.

\section{RESULTS}

GTG banding of 100 metaphases and corresponding FISH signals were analyzed at 8 months of age. After 5 years and 4 months, the research group contacted the parents of the child for follow-up testing using a chromosomal microarray analysis (CMA) approach that had become available in Central Brazil.

The karyotype was a constitutive 46,XX,r(13)[77]/45,XX,-13[17]/46,XX,idic r(13) [6] (Figure 1). Interphase and metaphase FISH analyses also showed the absence of 13qter (green spectrum) and the presence of 13q14.3 (red spectrum) in cells with ring chromosome 13 (Figure 2). An isodicentric chromosome was observed and confirmed by gray scale images on reverse 4,6-diamino-2-phenyl-indole staining, a tool included with the ISIS ${ }^{\circledR}$ software (Metasystems Corporation) to highlight centromeres. The ring chromosomes showed 2 red signals for the region 13q14.3 confirming the presence of chromosome 13 (see Figure 2). Chromosome microarray analysis detected $15.39 \mathrm{Mb}$ deletions at 13q32.3-q34 $\operatorname{arr}[\mathrm{hg} 19]$ 13q3 2.3q34(99,712,845-115,107,733)x1 (Figure 3). This deletion involved the following genes: 
DOCK9, UBAC2-AS1, UBAC2, MIR548AN, GPR18, GPR183, FKSG29, MIR623, TM9SF2, CLYBL, MIR4306, ZIC5, ZIC2, PCCA, PCCA-AS1, A2LD1, TMTC4, NALCN-AS1, NALCN, ITGBL1, FGF14, MIR2681, MIR4705, FGF14-IT1, LOC283481, TPP2, METTL21C, CCDC168, TEX30, KDELC1, BIVM, BIVM-ERCC5, ERCC5, METTL21CP1, SLC10A2, MIR548AS, DAOA-AS1, DAOA, LINC00343, LINC00460, EFNB2, ARGLU1, LINC00551, LINC00443, FAM155A, LIG4, ABHD13, TNFSF13B, MYO16, MYO16-AS1, IRS2, COL4A1, COL4A2, COL4A2-AS1, RAB20, CARKD, CARS2, ING1, LINC00346, ANKRD10, ARHGEF7, TEX29, SOX1, SPACA7, TUBGCP3, C13orf35, ATP11A, MCF2L-AS1, MCF2L, F7, F10, PROZ, PCID2, CUL4A, LAMP1, GRTP1, ADPRHL1, DCUN1D2, TMCO3, TFDP1, ATP4B, GRK1, LINC00552, FAM70B, GAS6-AS1, GAS6, LOC100506394, LINC00452, LINC00565, RASA3, CDC16, UPF3A, and CHAMP1. The karyotypes and CMA analyses of both parents were unremarkable.

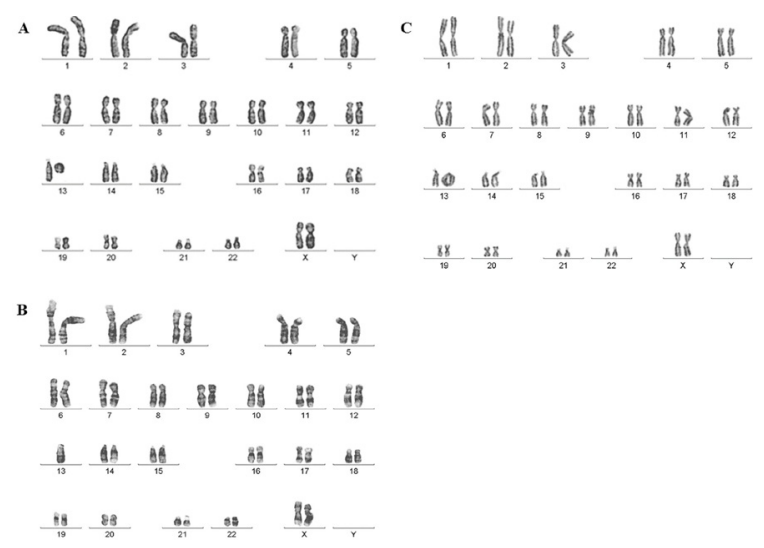

Figure 1. G-banded karyotype of the proband. A. Ring chromosome 13, B. monosomy of chromosome 13, and C. isodicentric ring chromosome 13.

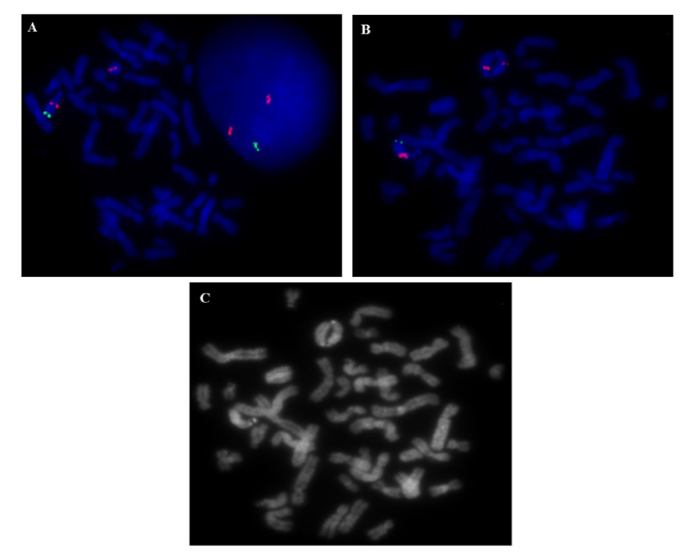

Figure 2. Fluorescence in situ hybridization analyses on peripheral lymphocytes. A. Absence of the 13qter-specific probe signal (green spectrum) and the presence of 13q14.3-specific probe signal (red spectrum) on the r(13) in the metaphase and interphase cell. B. Presence of 13q14.3-specific probe signal (red spectrum) and the absence of 13 qter-specific probe signal on the idic r(13). C. Isodicentric ring chromosome 13 defined by gray scale on reverse 4,6-diamino-2-phenyl-indole staining. 


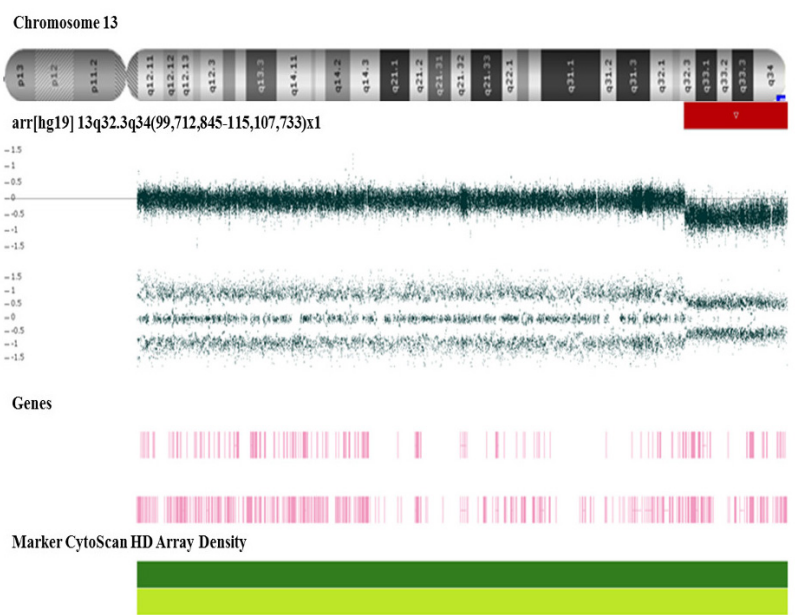

Figure 3. Chromosomal microarray analyses. Copy number variation is shown by $15.39-\mathrm{Mb}$ deletions at chromosome bands 13q32.3-q34 (red dots).

\section{DISCUSSION}

This report presents the first case of a child from Central Brazil harboring alterations of chromosome 13. The GTG-banding karyotype showed constitutive $r(13)$ in $77 \%$ of the cells, isodicentric 13 in $6 \%$ of the cells, and monosomy 13 in $17 \%$. Both monosomy 13 and isodicentric 13 were confirmed with FISH, which was also useful in determining the deletion of the terminal end of the long arm of chromosome 13 in the ring formation. However, banding karyotyping and FISH were unable to define the precise breakpoint of the terminal deletion. It was evident that the ring chromosome led to a partial deletion of the long arm of chromosome 13. Thus, based on probe density, high-resolution CMA allowed refinement of the breakpoint region of the affected chromosome 13 and was useful in determining the gene content within the deleted region. However, due to the nature of the chromosomal rearrangements, CMA identified only the microdeletion that comprised the majority of the mosaicism in our proband. Gain of isodicentric chromosome 13 and monosomy 13 were absent in the CMA due to its limitations for detecting low-level mosaicism.

Herein we report the first postnatal case of chromosome 13 with only $r(13)$, isodicentric 13, and monosomy 13. Our findings are similar to those of a report on a prenatal product of a pregnancy in which the parents elected to terminate at 23 weeks of gestation (Chen et al., 2013). The proband had a deletion of $15.39 \mathrm{Mb}$ involving 13q32, which is considered the critical band for the most severe phenotypes in $13 q$ deletions syndrome.

Haploinsufficiency of the genes within the deleted region is the most probable cause of the proband phenotype. Brown et al. (1993) defined 13q32 as the critical region for the most severe phenotypes, showing malformations in the brain, eyes, distal limbs, and genitourinary and gastrointestinal tracts, severe mental retardation, and short stature. The previous description is in agreement with our findings. Thus, $13 \mathrm{q} 32$ deletions describe a complex phenotype related mainly to brain, eye, and urinary tract malformations and severe mental retardation. Ballarati et al. (2007) defined a minimal deletion interval associated with DWM to the 13q32.2-33.2 region and Gul et al. (2005) reported a 13q31.2/32.1-qter deletion in a fetus with 
DWM. In this context, the proband exhibited the traits of $13 \mathrm{q} 32$ deletion syndrome including DWM in accordance with previous reports.

Herein we contrast the phenotypic findings of our proband with Online Mendelian Inheritance in Man (OMIM) morbid genes. The present case was characterized by haploinsufficiency of ZIC5 and ZIC2. Mutations in ZIC2 have been associated with a causality mechanism of holoprosencephaly (HPE) and may contribute to the severe brain malformations of patients with del(13q) (Brown et al., 1998). Our data corroborate this finding as magnetic nuclear resonance image of the proband indicated HPE. Moreover, research has implicated ZIC 2 deletions in $2 \%$ of HPE cases (Bendavid et al., 2006; 2007). Mutations of ZIC2 are frequently de novo and $70 \%$ of cases are due to deletions (Ribeiro et al., 2012), a finding that also support our results. Additional studies have indicated that ZIC2 has a pleiotropic effect and variable penetrance that may be the result of a loss of contiguous genes that in turn may influence gene expression (Mademont-Soler et al., 2010).

The proband had severe eye malformations. In addition to this phenotypic observation, the haploinsufficiency of EFNB2 has been proposed as a candidate gene for congenital eye malformations (Ballarati et al., 2007) and may be related to urorectal development and genital malformations in males (Walczak-Sztulpa et al., 2008). EFNB2 encodes a member of the ephrin family with specificity for ephrin receptors, which, in animal models, participate in several aspects of visual system development (Williams et al., 2003). Controversies remain about which genes are involved in the malformation of the ocular system. A set of changes in the eyes is a phenotypic trait often observed in individuals with 13q32 deletion and other genes in this region may also participate in the development of the phenotype. Additional studies will be needed to corroborate or refute this hypothesis. The deletion presented in our report confirms the importance of investigating genes in the 13q32.3-q34 region to identify those that may be involved in eye malformation.

We also found reports implicating the haploinsufficiency of SOX1 and ARHGEF7 in the development of the brain and cerebral cortex. These genes may also be responsible for microcephaly and cortical development (Kirchhoff et al., 2009). Deletion of COL4A1 has been associated with congenital anomalies of the eyes, brain, and kidneys in mice. Moreover, deletions of COL4A1 were first shown to cause porencephaly, cerebral hemorrhage, and microangiopathy in humans (Rodahl et al., 2013). Heterozygous mutations of COL4A2 have been associated with porencephaly 2 (OMIM 614483) (Yoneda et al., 2012) and increased nuchal translucency (Jeanne et al., 2012; Weng et al., 2012). Haploinsufficiency of COL4A1 and COL4A2 may be associated with susceptibility to intracerebral hemorrhage (OMIM 614519) (Jeanne et al., 2012; Weng et al., 2012) and increased nuchal translucency (Chen et al., 2012).

Moreover, F7 and $F 10$ have been associated with factor VII (Carew et al., 2000) and factor X deficiencies (Zhou et al., 2013), respectively. Coagulation deficiencies were not investigated in the proband. Furthermore, the haploinsufficiency of ITGBL1 is associated with lung hypoplasia (Berg et al., 1999). No evidence of lung defects was found in our proband.

The current report of a girl with 13q32.3-q34 deletion associated with DWM and a complex phenotype is intended to help in the characterization of rare and variable phenotypes associated with chromosome 13 deletions. Karyotype-phenotype correlations will facilitate future investigations of the haploinsufficiency of specific genes. However, due to the rarity of cases, studies must be carried out as part of an international consortium. Thus, the current case report is intended to act as the registry of a rare case of chromosomal rearrangement involving chromosome 13 in Central Brazil. Further studies are needed to define whether genetic 
haploinsufficiency is associated with each major 13q deletion anomaly or whether one or more putative genes of the critical regions are contributing to these congenital malformations. Taken together, the data reported herein and those from the literature allow for an update of the genotype-phenotype map of the 13q32.3-q34 chromosome segment.

\section{ACKNOWLEDGMENTS}

We thank Dr. Rinaldo Wellerson Pereira from Universidade Católica de Brasília for his assistance and for coordinating our ExeGenS "Rede de Excelência em Genética e Genômica Molecular Aplicada a Saúde Humana". We are also grateful to the proband and her family for participating in this investigation.

\section{REFERENCES}

Ballarati L, Rossi E, Bonati MT, Gimelli S, et al. (2007). 13q Deletion and central nervous system anomalies: further insights from karyotype-phenotype analyses of 14 patients. J. Med. Genet. 44: e60.

Bendavid C, Dubourg C, Gicquel I, Pasquier L, et al. (2006). Molecular evaluation of foetuses with holoprosencephaly shows high incidence of microdeletions in the HPE genes. Hum. Genet. 119: 1-8.

Bendavid C, Dubourg C, Pasquier L, Gicquel I, et al. (2007). MLPA screening reveals novel subtelomeric rearrangements in holoprosencephaly. Hum. Mutat. 28: 1189-1197.

Berg RW, Leung E, Gough S, Morris C, et al. (1999). Cloning and characterization of a novel beta integrin-related cDNA coding for the protein TIED ("ten beta integrin EGF-like repeat domains") that maps to chromosome band 13q33: A divergent stand-alone integrin stalk structure. Genomics 56: 169-178.

Brown S, Gersen S, Anyane-Yeboa K and Warburton D (1993). Preliminary definition of a "critical region" of chromosome 13 in q32: report of 14 cases with 13q deletions and review of the literature. Am. J. Med. Genet. 45: 52-59.

Brown SA, Warburton D, Brown LY, Yu CY, et al. (1998). Holoprosencephaly due to mutations in ZIC2, a homologue of Drosophila odd-paired. Nat. Genet. 20: 180-183.

Carew JA, Pollak ES, Lopaciuk S and Bauer KA (2000). A new mutation in the HNF4 binding region of the factor VII promoter in a patient with severe factor VII deficiency. Blood 96: 4370-4372.

Chen CP, Tsai CH, Chern SR, Wu PS, et al. (2013). Prenatal diagnosis and molecular cytogenetic characterization of mosaic ring chromosome 13. Gene 529: 163-168.

Chen JL, Yang YF, Huang C, Wang J, et al. (2012). Clinical and molecular delineation of 16p13.3 duplication in a patient with congenital heart defect and multiple congenital anomalies. Am. J. Med. Genet. A 158A: 685-688.

Gul A, Cebeci A, Erol O, Ceylan Y, et al. (2005). Prenatal diagnosis of 13q-syndrome in a fetus with Dandy-Walker malformation. Obstet. Gynecol. 105: 1227-1229.

Huang C, Yang YF, Yin N, Chen JL, et al. (2012). Congenital heart defect and mental retardation in a patient with a 13q33.1-34 deletion. Gene 498: 308-310.

Jeanne M, Labelle-Dumais C, Jorgensen J, Kauffman WB, et al. (2012). COL4A2 mutations impair COL4A1 and COL4A2 secretion and cause hemorrhagic stroke. Am. J. Hum. Genet. 90: 91-101.

Kirchhoff M, Bisgaard AM, Stoeva R, Dimitrov B, et al. (2009). Phenotype and 244k array-CGH characterization of chromosome 13q deletions: an update of the phenotypic map of 13q21.1-qter. Am. J. Med. Genet. A 149A: 894-905.

Lele KP, Penrose LS and Stallard HB (1963). Chromosome deletion in a case of retinoblastoma. Ann. Hum. Genet. 27: 171-174.

Mademont-Soler I, Morales C, Armengol L, Soler A, et al. (2010). Description of the smallest critical region for DandyWalker malformation in chromosome 13 in a girl with a cryptic deletion related to t(6;13)(q23;q32). Am. J. Med. Genet. A 152A: 2308-2312.

Ribeiro LA, Roessler E, Hu P, Pineda-Alvarez DE, et al. (2012). Comparison of mutation findings in ZIC2 between microform and classical holoprosencephaly in a Brazilian cohort. Birth Defects Res. A Clin. Mol. Teratol. 94: 912-917.

Rodahl E, Knappskog PM, Majewski J, Johansson S, et al. (2013). Variants of anterior segment dysgenesis and cerebral involvement in a large family with a novel COL4A1 mutation. Am. J. Ophthalmol. 155: 946-953.

Uwineza A, Pierquin G, Gaillez S, Jamar M, et al. (2013). Clinical, cytogenetic and molecular characterization of two cases of mosaic ring chromosome 13. Genet. Couns. 24: 193-200.

Valdes-Miranda JM, Soto-Alvarez JR, Toral-Lopez J, González-Huerta L, et al. (2014). A novel microdeletion involving 
the 13q31.3-q32.1 region in a patient with normal intelligence. Eur. J. Med. Genet. 57: 60-64.

Verma RS and Babu A (1995). Human chromosomes principles and techiniques. 2nd edn. McGraw-Hill, New York.

Walczak-Sztulpa J, Wisniewska M, Latos-Bielenska A, Linné M, et al. (2008). Chromosome deletions in 13q33-34: report of four patients and review of the literature. Am. J. Med. Genet. A 146A: 337-342.

Weng YC, Sonni A, Labelle-Dumais C, de LM, et al. (2012). COL4A1 mutations in patients with sporadic late-onset intracerebral hemorrhage. Ann. Neurol. 71: 470-477.

Williams SE, Mann F, Erskine L, Sakurai T, et al. (2003). Ephrin-B2 and EphB1 mediate retinal axon divergence at the optic chiasm. Neuron 39: 919-935.

Yoneda Y, Haginoya K, Arai H, Yamaoka S, et al. (2012). De novo and inherited mutations in COL4A2, encoding the type IV collagen alpha2 chain cause porencephaly. Am. J. Hum. Genet. 90: 86-90.

Zhou JW, Liang Q, Chen Q, Xie Y, et al. (2013). Molecular defects in the factor X gene caused by novel heterozygous mutations IVS5+1G>A and Asp409del. Haemophilia 19: 94-99. 